\title{
Computational Anatomy and Implicit Object Representation: A Level Set Approach
}

\author{
Wei-Hsun Liao ${ }^{1}$, Luminita Vese ${ }^{2}$, Sung-Cheng Huang ${ }^{1}$, \\ Marvin Bergsneider ${ }^{3}$, and Stanley Osher ${ }^{2}$ \\ ${ }^{1}$ Department of Biomathematics, UCLA David Geffen School of Medicine, LA, CA, 90095 \\ 2 Department of Mathematics, UCLA, LA, CA, 90095 \\ ${ }^{3}$ Brain Injury Center, UCLA, LA, CA, 90095
}

\begin{abstract}
In this paper, we look at the fundamental problem of object matching in computational anatomy. We present a new framework for warping pairs of overlapping and non-overlapping shapes, open curves, and landmarks based on the level set approach. When implemented in 3-D, the same framework could be used to warp 3-D objects with minimal modification. Our approach is to use the level set functions to represent the objects to be matched. Using this representation, the problem becomes an energy minimization problem. Cost functions for warping overlapping, non-overlapping, open curves, and landmarks are proposed. Euler-Lagrange equations are applied and gradient descent is used to solve the corresponding partial differential equations. Moreover, a general framework for linking the level set approach and the infinite dimensional group actions is discussed.
\end{abstract}

\section{Introduction}

Computational anatomy $[1,2]$ is an emerging new discipline that deals with analyzing and making sense of the large collection and database of brain imaging. A fundamental problem in computational anatomy is image warping, or dynamically mapping one brain dataset to another through diffeomorphic transformation. In this paper, we will focus on developing techniques for matching anatomically important objects.

Object warping is a challenging problem not only in computational anatomy but also in computer vision, pattern recognition as well as many other scientific fields. In the past decade, several strategies of non-rigid warping algorithms have been proposed that could be divided into two groups: landmark based and dense matching.

Landmark matching involves first identifying user-defined landmarks that need to be matched. By interpolating the discrete matching of the landmarks, one tries to obtain a dense diffeomorphism for the whole image. Dense matching starts by forming a cost function that is minimized when the objects are matched. In order to ensure smooth matching, a regularizing term on the deformation field is added.

In this paper, we will use the terms template and study to denote the images to be matched. Let us denote the template image as $T(x)$ and the study image as $S(x)$ which 
are images on the spatial domain $\Omega \subset R^{n}$. The problem of image warping is to find a displacement field $u(x)$ at each point $x$ such that a properly defined cost function, which will be denoted by $D(T, S, u)$, between the deformed template and the study is minimized. The displacement field is a vector field such that given any displacement field $u$ the deformed template is given by $T(x-u)$. The term displacement is used because it can be viewed as how a point in the template is moved away from its original location. The most common way to define the measure between the deformed template and the study image is based on the $L^{2}$ norm

$$
D(T, S, u)=\frac{1}{2} \int_{\Omega}|T(x-u)-S(x)|^{2} d x .
$$

Gradient descent of the corresponding Euler-Lagrange equation is often used to minimize this cost function

$$
\frac{\partial u(x, t)}{\partial t}=f(x, u(x, t)), f(x, u)=\left.[T(x-u)-S(x)] \bullet \nabla T\right|_{x-u} .
$$

The function $f(x, u)$, which is often called the force field or the body force, describes the derivative of the cost function with respect to the displacement field $u$.

\section{Previous Work}

Several models for regularizing the deformation field have been proposed. We will give an overview by looking at those with the most theoretical interests

\subsection{Hyper-elastic Matching}

In hyper-elastic matching [3], the authors tried to draw analogy between image warping and deforming elastic plates. Under the assumption of linear elasticity, which holds for relatively small displacement field only, we arrive at the following equation that should hold at equilibrium

$$
\mu \Delta u(x, t)+(\mu+\lambda) \nabla(\nabla \cdot u(x, t))=f(x, u(x, t)) .
$$

Here $\mu$ and $\lambda$ are the Lame constants. Due to this linear elasticity assumption, large-magnitude displacements are severely penalized and thus hyper-elastic model is not suitable for problems in which large and highly nonlinear deformation is needed.

\subsection{The Fluid Dynamic Approach}

An extension of the hyper-elastic matching, the viscous fluid matching, was proposed by Christensen [4-6] using the Navier-Stokes formulation. This approach can be viewed as the linear elastic operator applied to the velocity field, which is related to the displacement field in a standard Eulerian framework. The advantage of this method is that it allows large-magnitude deformations since stress constraining the deformation relaxes over time. 


\subsection{Large Deformation through Diffeomorphisms by Infinite Dimensional Group Actions}

In the past decade, many researchers have tried to establish rigorous theories based on continuum mechanics that ensure diffeomorphic transformation by working on the forward and inverse mapping directly (see [7] and the references therein).

In summary, Let $g^{-1}(x)=x-u$ be the deformation field, and $G$ be the group which is formed by all the diffeomorphisms that map $\Omega \in X$ to itself. A path in $G$

$$
g(x, t)=g_{t}(x), g_{t} \in G \text { for } \forall t \in[0,1] .
$$

is linked to the velocity field $v_{t}$ by the following equation

$$
\frac{\partial}{\partial t} g_{t}(x)=v_{t}\left(g_{t}(x)\right) \text {. }
$$

Once the forward path $g_{t}$ is defined, the inverse path is uniquely determined by

$$
g_{t}^{-1}\left(g_{t}(x)\right)=x
$$

and is linked to the velocity by

$$
\frac{\partial}{\partial t} g_{t}^{-1}(x)=-D g_{t}^{-1}(x) v_{t}(x) \text {. }
$$

Given a path $g(x, t)$ and its associated velocity, we define the energy of the path by

$$
E(g)=\int_{t=0}^{1}\left\|v_{t}(x)\right\|_{L}^{2} d t=\int_{t=0}^{1}\left\langle L v_{t}(x), L v_{t}(x)\right\rangle d t .
$$

Here $L$ is a differential operator. Furthermore, let us define the momentum $p$ by

$$
p(x, t)=p_{t}(x)=L^{\dagger} L v_{t}(x) .
$$

Here $L^{\dagger}$ is the adjoint operator of $L$. It can be shown that diffeomorphisms could be ensured under mild restrictions on the operator $L$ (see [8]). We then have the following theorem which could be found in $[7,9]$.

\section{Theorem 1}

Let $g_{0}$ and $g_{1}$ be elements in $\mathrm{G}$, the function $d$ defined as

$$
d\left(g_{0}, g_{1}\right)=\left(\inf _{\frac{\partial}{\partial t} g_{t}^{-1}=-D g_{t}^{-1} v_{t}: g_{t=0}=g_{0}, g_{t=1}=g_{1}}^{1} \int_{t=0}^{1}\left\|v_{t}(x)\right\|_{L}^{2} d t\right)^{\frac{1}{2}}
$$

is a metric on $G$. Moreover, the geodesic satisfies the Euler-Lagrange equation 


$$
\frac{d p_{t}}{d t}+\left(v_{t} \bullet \nabla\right) p_{t}+\operatorname{div}\left(v_{t}\right) p_{t}+\sum_{i} \nabla\left(\left(v_{t}\right)_{i}\right)\left(p_{t}\right)_{i}=0 .
$$

Following [7], the operator $L$ used in this paper will be in the following form

$$
L=(-a \Delta+b I)^{m}, a>0, b>0, m \in \mathbb{N} .
$$

\section{Theory}

In this paper, we try to generalize the landmark matching problem by replacing the landmarks with objects of different types. We turn to the idea of level set method for ways of representing objects. The level set method was first proposed in [10] and has been proven to be a powerful tool in front tracking as well as many other applications. For an overview on level set method, we refer the readers to [11] and the references therein.

\subsection{Shape Matching}

Let us start with shape matching. A shape could be represented by a level set function with the boundary of the shape being the zero level curve of the level set function (positive value inside the shape and negative outside). Throughout this paper we will use the following notation. The shapes in the template image will be denoted by the level set functions $\varphi_{r}, \varphi_{2}, \ldots, \varphi_{n}$ and the corresponding shapes in the study image by $\psi_{r}$, $\psi_{2}, \ldots, \psi_{n}$.

\section{One Pair of Overlapping Shapes}

In order to derive a suitable cost function that is always non-negative and only takes the value zero when the two level set functions match, we minimize the symmetric difference of the two level set functions (see $[12,13]$ for similar approaches).

$$
D_{\text {over }}(T, S, t)=\int_{\Omega} H(\psi(x))\left[1-H\left(\varphi\left(g_{t}^{-1}\right)\right)\right] d x+\int_{\Omega} H\left(\varphi\left(g_{t}^{-1}\right)\right)[1-H(\psi(x))] d x .
$$

The force field of this cost function is

$$
f_{\text {over }}(x, t)=[1-2 H(\psi(x))] \delta\left(\varphi\left(g_{t}^{-1}\right)\right) \nabla \varphi\left(g_{t}^{-1}\right) .
$$

Here $H$ and $\delta$ are the Heaviside and the delta function.

\section{One Pair of Non-overlapping Shapes}

The above cost function does not work for non-overlapping shapes. The reason is that by minimizing the cost function, $\varphi$ will simply shrink to a point and the cost function will reach a local minimum. To overcome this, we integrate $-\psi$ in the area $\varphi>0, \psi<0$, and integrate $-\varphi$ in the $\varphi<0, \psi>0$ and we now have to initialize the level set functions to be the signed distance function to their zero level sets. 


$$
\begin{aligned}
& D_{\text {nonoverlapping }}(T, S, t)=\int_{\Omega}-\varphi\left(g_{t}^{-1}\right) H(\psi(x))\left[1-H\left(\varphi\left(g_{t}^{-1}\right)\right)\right] d x \\
& +\int_{\Omega}-\psi(x) H\left(\varphi\left(g_{t}^{-1}\right)\right)[1-H(\psi(x))] d x .
\end{aligned}
$$

In this case the force field is given by

$$
\begin{aligned}
& f_{\text {nonoverlapping }}(x, t)=-\left\{H ( \psi ( x ) ) \left[1-H\left(\varphi\left(g_{t}^{-1}\right)\right)\right.\right. \\
& \left.\left.-\varphi\left(g_{t}^{-1}\right) \delta\left(\varphi\left(g_{t}^{-1}\right)\right)\right]+[1-H(\psi(x))] \psi(x) \delta\left(\varphi\left(g_{t}^{-1}\right)\right)\right\} \nabla \varphi\left(g_{t}^{-1}\right) .
\end{aligned}
$$

\subsection{Geodesic Open Curve Matching}

Our task is to find a cost function between an open curve $C$ in the template and another open curve $C^{\prime}$ in the study. We follow the idea in [14] by extending $C$ to a closed curve (represented by the zero level set of $\varphi_{1}$ ), and we further draw a second closed curve (represented by the zero level set of $\varphi_{2}$ ), which crosses the zero level set of $\varphi_{1}$ only at the two end points of $C$. Then the open curve $C$ is represented by

$$
C=\left\{x \mid \varphi_{1}(x)=0 \text { and } \varphi_{2}(x)>0\right\} .
$$

The open curve $C^{\prime}$ in the study image could also be represented by two level set functions $\psi_{1}$ and $\psi_{2}$. Let us further denote the distance functions of $C$ and $C$ ' by $D_{\mathrm{s}}(\mathrm{x})$ and $D_{\mathrm{T}}(\mathrm{x})$. We then reach at the following cost function

$$
\begin{aligned}
& D_{\text {curve }}(T, S, t)=\int_{\Omega} D_{s}(x) \delta\left(\varphi_{1}\left(g_{t}^{-1}\right)\right)\left|\nabla \varphi_{1}\left(g_{t}^{-1}\right)\right| H\left(\varphi_{2}\left(g_{t}^{-1}\right)\right) d x \\
& +\int_{\Omega} D_{T}\left(g_{t}^{-1}\right) \delta\left(\psi_{1}(x)\right)\left|\nabla \psi_{1}(x)\right| H\left(\psi_{2}(x)\right) d x .
\end{aligned}
$$

This cost function could be viewed as the sum of two line segment integrals with respect to the other curve's distance function. It can be shown that (for derivations, please refer to [15]) in this case, the force field is

$$
\begin{aligned}
& f_{\text {curve }}(x, t)=-\frac{\delta_{1}}{\left|\nabla \varphi_{1}\left(g_{t}^{-1}\right)\right|}\left\{H_{2}<\nabla D_{s}, \nabla \varphi_{1}\left(g_{t}^{-1}\right)>\right. \\
& \left.+D_{s} \delta_{2}<\nabla \varphi_{2}\left(g_{t}^{-1}\right), \nabla \varphi_{1}\left(g_{t}^{-1}\right)>\right\} \nabla \varphi_{1}\left(g_{t}^{-1}\right) \\
& -\delta_{1} D_{s} H_{2} \operatorname{div}\left(\frac{\nabla \varphi_{1}\left(g_{t}^{-1}\right)}{\left|\nabla \varphi_{1}\left(g_{t}^{-1}\right)\right|}\right) \nabla \varphi_{1}\left(g_{t}^{-1}\right) \\
& +D_{s} \delta_{1} \delta_{2}\left|\nabla \varphi_{1}\left(g_{t}^{-1}\right)\right| \nabla \varphi_{2}\left(g_{t}^{-1}\right)+\delta\left(\psi_{1}\right)\left|\nabla \psi_{1}\right| H\left(\psi_{2}\right) \nabla D_{T}\left(g_{t}^{-1}\right) .
\end{aligned}
$$

Here the following short notations are used 


$$
\delta_{1}=\delta\left(\varphi_{1}\left(g_{t}^{-1}\right)\right), H_{2}=H\left(\varphi_{2}\left(g_{t}^{-1}\right)\right), \text { and } \delta_{2}=\delta\left(\varphi_{2}\left(g_{t}^{-1}\right)\right)
$$

\subsection{Landmark Matching}

Landmark matching problem could also be formulated in the level set framework. Let the landmark in the template be the intersection of two level set functions $\varphi_{1}$ and $\varphi_{2}$, and $D_{T}$ be the distance function to the landmark. We define similarly the corresponding terms for the landmark in the study. Now consider the following cost function that recovers the sum of the distance between these two landmarks under the action of the forward and inverse mapping (see [16] for detailed discussions)

$$
\begin{aligned}
D_{\text {landmark }}(T, S, t)= & \int_{\Omega} D_{S} \delta\left(\varphi_{1}\left(g_{t}^{-1}\right)\right) \delta\left(\varphi_{2}\left(g_{t}^{-1}\right)\right)\left|\nabla \varphi_{1}\left(g_{t}^{-1}\right) \times \nabla \varphi_{2}\left(g_{t}^{-1}\right)\right| d x \\
& +\int_{\Omega} D_{T}\left(g_{t}^{-1}\right) \delta\left(\psi_{1}\right) \delta\left(\psi_{2}\right)\left|\nabla \psi_{1} \times \nabla \psi_{2}\right| d x
\end{aligned}
$$

With this cost function, the force field is then

$$
\begin{aligned}
& f_{\text {landmark }}(x, t)= \\
& -\operatorname{div}\left(\frac{P_{\nabla \varphi_{2}\left(g_{t}^{-1}\right)} \nabla \varphi_{1}\left(g_{t}^{-1}\right)}{\left|P_{\nabla \varphi_{2}\left(g_{t}^{-1}\right)} \nabla \varphi_{1}\left(g_{t}^{-1}\right)\right|} \nabla \varphi_{2}\left(g_{t}^{-1}\right) \mid D_{S}\right) \delta\left(\varphi_{1}\left(g_{t}^{-1}\right)\right) \delta\left(\varphi_{2}\left(g_{t}^{-1}\right)\right) \nabla \varphi_{1}\left(g_{t}^{-1}\right) \\
& -\operatorname{div}\left(\frac{P_{\nabla \varphi_{1}\left(g_{t}^{-1}\right)} \nabla \varphi_{2}\left(g_{t}^{-1}\right)}{\left|P_{\nabla \varphi_{1}\left(g_{t}^{-1}\right)} \nabla \varphi_{2}\left(g_{t}^{-1}\right)\right|}\left|\nabla \varphi_{1}\left(g_{t}^{-1}\right)\right| D_{S}\right) \delta\left(\varphi_{1}\left(g_{t}^{-1}\right)\right) \delta\left(\varphi_{2}\left(g_{t}^{-1}\right)\right) \nabla \varphi_{2}\left(g_{t}^{-1}\right) \\
& +\delta\left(\psi_{1}\right) \delta\left(\psi_{2}\right)\left|\nabla \psi_{1} \times \nabla \psi_{2}\right| \nabla D_{T}\left(g_{t}^{-1}\right)
\end{aligned}
$$

\section{Incorporating the Level Set Method and Diffeomorphisms Generated by Infinite Dimensional Group Actions}

\subsection{Level Set Based Matching through Infinite Dimensional Group Actions}

The level set based object matching could be integrated into the infinite dimensional group actions approach. Thus, we can examine the inexact and space time growth image matching problem as in theorem 4.1 and 4.2 of [7]. In the case of inexact matching problem, we have the following theorem

\section{Theorem 2 (Inexact Level Set Based Object Matching)}

The path that minimizes the following inexact matching problem

$$
\inf _{\frac{\partial}{\partial t} g_{t}^{-1}=-D g_{t}^{-1} v_{t}, g_{t=0}=i d} \int_{t=0}^{1}\left\|v_{t}(x)\right\|_{L}^{2} d t+D(T, S, t=1)
$$

satisfies (12) and the following boundary condition at $t=1$ 


$$
p(x, 1)-\left[f(x, 1)^{T} D g_{t=1}^{-1}\right]^{T}=0 .
$$

Here $D(T, S, t)$ and $f$ are the cost function and force field defined in section 3 . For proof, simply combine the arguments in [7] and [15].

\subsection{The Modified Beg's Algorithm}

In this section, we will describe how to solve the inexact matching problem in 4.1 (the space-time growth problem could be handled similarly). Our algorithm is a modified version of the algorithm proposed by Faisal Beg (see [7]) for solving inexact image matching via variations with respect to the velocity field. The advantage of this approach is that we obtain the forward and inverse transformation and thus it allows us to do distance function re-initialization as described in [15].

\section{(Modified Beg's) Algorithm for Inexact Level Set Based Object Matching}

Initialize $v^{\text {old }}=0$, choose a small number $\varepsilon$, for all $t$ in [0 1],

\section{Step1 (fixed point iteration)}

$$
\begin{aligned}
& \text { Solve } g_{t}^{\text {new }} \text { and } g_{t}^{-1 \text { new }} \text { by } \\
& \frac{d}{d t} g_{t}^{\text {new }}=v_{t}^{\text {old }}\left(g_{t}^{\text {new }}\right), \frac{d}{d t} g_{t}^{-1 \text { new }}(x)=-D g_{t}^{-1 \text { new }}(x) v_{t}^{\text {old }}(x) . \\
& \text { Compute } g_{1}^{\text {new }}\left(g_{t}^{-1 \text { new }}(\bullet)\right) .
\end{aligned}
$$

\section{Step2}

$$
\begin{aligned}
& v_{t}^{\text {new }}=v_{t}^{\text {old }}-\varepsilon \bullet \operatorname{grad} \\
& \operatorname{grad}=v_{t}^{\text {old }}-\left(L^{\dagger} L\right)^{-1}\left[\left|D g_{1}^{\text {new }}\left(g_{t}^{-1 \text { new }}\right)\right| \sum_{i} f_{i}\left(g_{1}^{\text {new }}\left(g_{t}^{-1 \text { new }}(x)\right), t=1\right)^{T} D g_{t}^{-1 \text { new }}\right]^{T} .
\end{aligned}
$$

Set $v_{t}^{\text {old }}=v_{t}^{\text {new }}$. Go back to step 1 until convergence.

Here the algorithm is presented in the most general case in which multiple objects are to be matched. Thus, the force field has several components $\left(f_{i}\right)$ and each component is defined as in section 3 depending on the nature of the object. Notice that in step 2 the gradient descent direction is in the sense of the operator $L^{+} L$ instead of the usual $L^{2}$ and thus could be interpreted as a smoothing step.

We could reinitialize the distance function once in several iterations by reinitializing the level set function under the action of $g_{t=1}^{-l}$ to its zero level set and compose again with $g_{t=1}$. To be more precise, we need to add the following step in 4.2 

Compute $\varphi_{i}^{*}(x) \triangleq \varphi_{i}\left(g_{t=1}^{-1 \text { new }}(x)\right)$
Reinitialize $\varphi_{i}^{*}(x)$ to its zero level set
Update $\varphi_{i} \leftarrow \varphi_{i}^{*}\left(g_{t=1}^{\text {new }}(x)\right)$
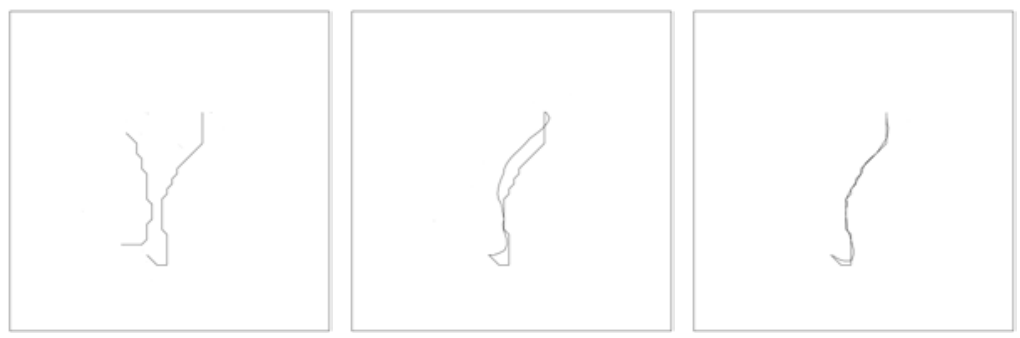

Fig. 1. Left panel: The two curves to be matched. Middle panel: The position of the curves after matching the end points. Right panel: The final result of matching the two curves.
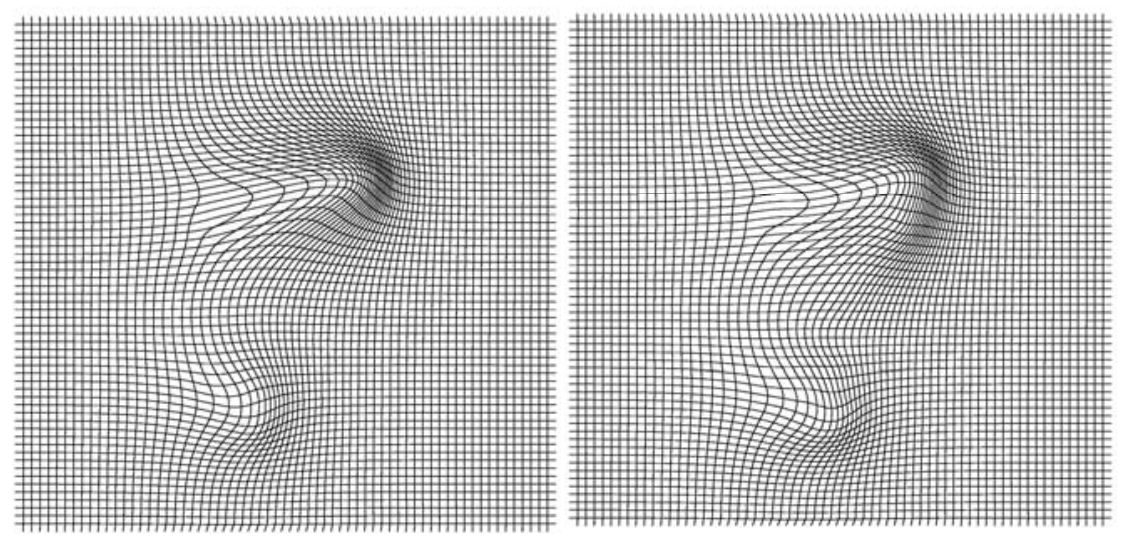

Fig. 2. Left panel: The corresponding deformation field of the middle panel in Figure 1. Right panel: The corresponding deformation field of the right panel in Figure 1.

\section{Results}

Open curve and landmark matching based on our method are presented (for shape matching, please refer to $[15,16])$. All images are in the unit square discretized to a 64 by 64 grid, and the modified Beg's algorithm is used. The differential operator $L^{+} L$ used in these two numerical examples is $50(-\Delta+i d)^{2}$, and Fourier technique is employed to calculate the inverse of the operator in step 2 of the modified Beg's algorithm. Level set functions and distance functions in the template are re-initialized as described in 4.2 once in every 100 iterations.

Figure 1 (left panel) shows the relative position of the two open curves. To test the level set based landmark matching, the proposed cost function (20) is used to match 
the endpoints of the two curves and the result is shown in the middle panel of Figure 1 . The corresponding deformation of the underlying grid (the geodesic path at $\mathrm{t}=$ 1 ) in this case is shown in the left panel of Figure 2. Notice that matching the endpoints alone does not ensure the matching of the whole curve. The right panel of Figure 1 shows the matching of the whole curve by minimizing cost function (18), with the corresponding deformation (the geodesic path at $t=1$ ) shown in the right panel of Figure 2. For comparison, Figure 3 shows the geodesic path in these two examples at time $t=0.5$. The length of the geodesic path is 8.97 in the case of endpoint matching, and 10.78 in the case of whole curve matching.

This paper was partially supported by NIH grant NS30308 and P20MH65166.
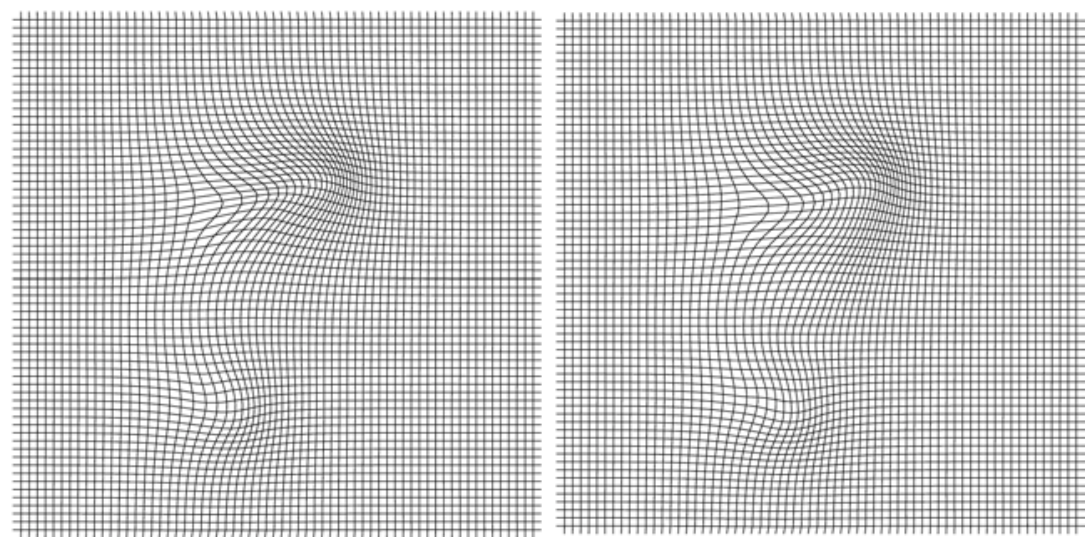

Fig. 3. Left panel: The corresponding geodesic path at $t=0.5$ of the middle panel in Figure 1 . Right panel: The corresponding geodesic path at $t=0.5$ of the right panel in Figure 1.

\section{References}

1. U. Grenander and M. I. Miller, "Computational anatomy: An emerging discipline," Quarterly of Applied Mathematics, vol. 56, pp. 617-694, 1998.

2. P. Thompson and A. W. Toga, "A framework for computational anatomy," Computing and Visualization in Science, vol. 5, pp. 13-34, 2002.

3. R. Bajcsy and S. Kovacic, "Multiresolution Elastic Matching," Computer Vision Graphics and Image Processing, vol. 46, pp. 1-21, 1989.

4. G. E. Christensen, R. D. Rabbitt, and M. I. Miller, "Deformable templates using large deformation kinematics," IEEE Transactions on Image Processing, vol. 5, pp. 1435-47, 1996.

5. G. E. Christensen, R. D. Rabbitt, and M. I. Miller, "3D brain mapping using a deformable neuroanatomy," 1994.

6. G. E. Christensen, S. C. Joshi, and M. I. Miller, "Volumetric transformation of brain anatomy," IEEE Transactions on Medical Imaging, vol. 16, pp. 864-77, 1997.

7. M. I. Miller, A. Trouve, and L. Younes, "On the metrics and Euler-Lagrange equations of computational anatomy," Annual Review of Biomedical Engineering, vol. 4, pp. 375-405, 2002. 
8. P. Dupuis, U. Grenander, and M. I. Miller, "Variational problems on flows of diffeomorphisms for image matching," Quarterly of Applied Mathematics, vol. 56, pp. 587-600, 1998.

9. D. Mumford, "Pattern Theory: the Mathematics of Perception," in ICM 2002, vol. 3, 2002.

10. S. Osher and J. A. Sethian, "Fronts Propagating with Curvature-Dependent Speed - Algorithms Based on Hamilton-Jacobi Formulations," Journal of Computational Physics, vol. 79, pp. 12-49, 1988.

11. S. Osher and R. P. Fedkiw, "Level set methods: An overview and some recent results," Journal of Computational Physics, vol. 169, pp. 463-502, 2001.

12. R. T. Whitaker, "A level-set approach to image blending," IEEE Transactions on Image Processing, vol. 9, pp. 1849-61, 2000.

13. N. Paragios, M. Rousson, and V. Ramesh, "Matching Distance Functions: A Shape-to-Area Variational Approach for Global-to-Local Registration," ECCV, 2002.

14. P. Smereka, "Spiral crystal growth," Physica D, vol. 138, pp. 282-301, 2000.

15. W. H. Liao, A. Jhuu, M. Bergsneider, L. A. Vese, S. C. Huang, and S. Osher, "From Landmark Matching to Shape and Open Curve Matching: A Level Set Approach," in UCLA CAM report, 2002.

16. W. H. Liao, "Mathematical techniques in object matching and computational anatomy: a new framework based on the level set method," in Biomathematics. Los Angeles: UCLA, 2003. 